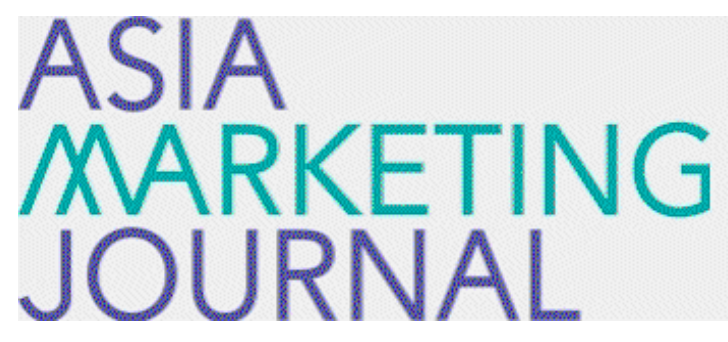

ASIA MARKETING JOURNAL

Volume 17 | Issue 2

Article 1

7-31-2015

\title{
Advertising Distinctiveness and Consumer Memory in Competitive Ad Environments
}

Ryosuke Takeuchi

Follow this and additional works at: https://amj.kma.re.kr/journal

Part of the Marketing Commons

\section{Recommended Citation}

Takeuchi, Ryosuke (2015) "Advertising Distinctiveness and Consumer Memory in Competitive Ad Environments," Asia Marketing Journal: Vol. 17 : Iss. 2 , Article 1.

Available at: https://doi.org/10.15830/amj.2015.17.2.1

This Article is brought to you for free and open access by Asia Marketing Journal. It has been accepted for inclusion in Asia Marketing Journal by an authorized editor of Asia Marketing Journal. 


\section{Advertising Distinctiveness and Consumer Memory in Competitive Ad Environments*}

Ryosuke Takeuchi**

It is becoming increasingly difficult for advertisers to reach target consumers because memory for an ad is inhibited in the presence of competing ads. While previous studies have focused on ad repetition as a factor that can reduce competitive interference, this study focuses on ad distinctiveness. We compare the effects of ad distinctiveness on recall for the ad and its competing ad in high competitive ad environments with those in relatively low competitive ad environments. Regarding the effect of ad distinctiveness on recall for the focal ad, the results show that when an advertiser makes its ad distinctive, recall for its own ad is enhanced in both high and low competitive ad environments although the positive effect is relatively weak in high competitive environments. The results also show that, regarding the effect of ad distinctiveness on recall for its competing ad, when an advertiser makes its ad distinctive, recall for its competing ad is enhanced in low competitive ad environments rather than in high competitive ad environments.

Key words: competitive ad environments, ad distinctiveness, memory, competitive interference

\section{Introduction}

Today, an average consumer is exposed to between 254 and 5,000 commercial messages per day (Creamer 2007) and, thus, "competitive interference" may occur, which is defined as impaired ability to remember information from an ad when it is similar to information from competing ads stored in memory (cf., Anderson and Neely 1996, p.237). Competitive interference has been considered as one of the great concerns of the advertising industry because it leads to higher communication costs to reach

\footnotetext{
* This work was supported by JSPS KAKENHI Grant Number 15J08649.

** Doctoral Researcher, Graduate School of Business and Commerce, Keio University (r.takeuchi@keio.jp)

The author thanks Prof. Akinori Ono for his suggestions and encouragements in all stages of this research.

The author also thanks the editor and three anonymous AMJ reviewers for their guidance.
} 
target consumers than ever before (Chunovic 2003: Unnava and Sirdeshmukh 1994).

Burke and Srull (1988) found that recall for an ad is inhibited by its competing ads. Since then, advertising researchers have regarded ad repetition as the most common factor that can reduce competitive interference (e.g., Burke and Srull 1988: Laroche, Cleveland, and Maravelakis 2006: Malaviya, Meyers-Levy, and Sternthal 1999: Yaveroglu and Donthu 2008). However, it is necessary to examine other factors because it has been found that ad repetition does not enhance recall for the ad in high competitive ad environments (Burke and Srull 1988; Malaviya et al. 1999).

Regarding the problem, some researchers have focused on ad distinctiveness - the extent to which an ad differs from its competing ads (cf., Guerard, Neath, Surprenant, and Tremblay 2010, p.83). They found that ad distinctiveness enhances recall for the ad (Keller 1991; Unnava and Sirdeshmukh 1994). That is, recall for an ad becomes higher when the advertiser makes its ad distinctive. However, previous studies have not considered explanatory and explained variables that need to be examined to fully understand consumer memory in competitive ad environments: (1) the interaction between ad distinctiveness (i.e., distinctive vs. undistinctive) and competition level (i.e., high vs. low) as an explanatory variable and (2) recall for a competing ad as an explained variable. Thus, in order to address these problems, we compare the effects of ad distinctiveness on memory for the focal ad and its competing ad in high competitive ad environments with those in low competitive ad environments.

\section{Theoretical Background}

\subsection{Competitive Interference}

An early study found that memory for an ad can be inhibited by retroactive/proactive interferences when subsequent/prior learning can interfere with one's ability to remember old/ new information (Blankenship and Whitely 1941; McKinney 1935).

Advertising researchers have employed the concept of interference as "competitive interference" when they have investigated recall for an ad in the presence of competing ads. Burke and Srull (1988) is, to our knowledge, the first research that explained the processes underlying competitive interference. According to Burke and Srull, when consumers are exposed to many ads in the same product category, they are more likely to confuse information from an ad with that from its competing ads in the same product category because information from similar ads is likely to be stored closely with each other in memory. Thus, it has been claimed that recall for an ad is inhibited by competing ads. Burke and Srull (1988) and the following studies 
have provided evidences that competitive interference may occur in print ads (Burke and Srull 1988), radio ads (Hammer, Riebe, and Kennedy 2009: Riebe and Dawes 2006), television ads (Jeong, Kim, and Zhao 2011), and ads in social media (Nelson-Field, Riebe, and Sharp 2013).

While these studies have investigated the effects of competitive interference on memory, Danaher, Bonfrer, and Dhar (2008) investigated the effects of competitive interference on sales. The results showed that competitive interference caused a serious decrease in sales of the focal brand as well as advertising elasticity of the brand.

\subsection{Ad Distinctiveness}

Ad distinctiveness plays an important role as a factor that can reduce competitive interference. Keller (1991) examined the effects of valence (persuasiveness and likability, p.465) on recall for an ad. According to Keller, when the level of valence for an ad is the same as those of valence for its competing ads, information from the focal ad may be stored closely with information from its competing ads in memory. In contrast, when the level of valence for an $\mathrm{ad}$ is different from those of valence for its competing ads, information from the focal ad may not be stored closely with information from its competing ads in memory. It may be easier for consumers to distinguish between information from the ad and information from its competing ads in the latter case. Therefore, Keller concluded that the level of recall for an ad is higher when the level of valence for the ad is different from those for its competing ads rather than when these ads are the same in terms of the level of valence.

Unnava and Sirdeshmukh (1994) examined the effects of modality (input sense that is activated by ad information, p.406) on recall for an ad. Assuming that there are two ads in a product category, they argued that when an $\mathrm{ad}$ is presented in the same modality as the other ad (e.g., both ads are print ads), the modality cues associated with these two kinds of ads are similar (e.g., both are visual). In contrast, when an ad is presented in different modality from the other ad (e.g., one is a print $\mathrm{ad}$, while the other is a radio ad), the modality cues associated with these two kinds of ads are different (e.g., one is visual, while the other is auditory). Distinctiveness of these modality cues may help consumers precisely retrieve information from an ad. Therefore, Unnava and Sirdeshmukh concluded that the level of recall for an ad is higher when the ad is presented in different modality from its competing ads than when the ad is presented in the same modality as its competing ads. 


\section{Hypotheses}

\subsection{The Effect of Ad Distinctiveness on Recall for the Focal Ad}

As mentioned in the previous section, it has been found that ad distinctiveness enhances recall for the ad (Keller 1991; Unnava and Sirdeshmukh 1994). However, it remains unclear whether competition level may affect the effect of ad distinctiveness on recall for the ad.

In high competitive ad environments, in which consumers are exposed to multiple competing ads, information from an ad would be separately stored with information from its competing ads in memory if the advertiser makes the ad distinctive. Nevertheless, in such environments, it may not be easy for consumers to recall information from the ad because considerable information from many competing ads is also stored in memory.

On the other hand, in relatively low competitive ad environments, in which consumers are $\mathrm{ex}^{-}$ posed to a single competing ad, they may easily recall information from an ad because little information from its competing ad is stored in memory. Thus, the following hypothesis is proposed.

H1: The positive effect of ad distinctiveness on recall for the ad is higher in low competitive ad environments than in high competitive ad environments.

\subsection{The Effect of Ad Distinctiveness on Recall for Any Competing Ad}

According to Jacoby and Craik (1979), distinctiveness of an item is always relative to its background. Therefore, when competitors make their ads distinctive, the focal ad may also become distinctive even if the advertiser does not try to make the ad distinctive. Conversely, when an advertiser makes its ad distinctive, its competing ads may also become distinctive even if the competitors do not try to make their ads distinctive. These phenomena are more likely to occur in low competitive ad environments than in high competitive ad environments.

In high competitive ad environments, in which consumers are exposed to multiple competing ads, any competing ad does not become distinctive because it may be still similar to the other ads in the same product category if an advertiser makes its ad distinctive. As a result, information from any competing ad would be separately stored with information from the focal ad, but closely stored with information from the other ads in memory. In such environments, consumers may hardly recall information from any competing ad because they are more likely to confuse it with the other ads.

On the other hand, in relatively low competitive ad environments, in which consumers are exposed to a single competing ad, any 
competing ad also becomes distinctive because there are no other ads that may be similar to the ad if an advertiser makes its ad distinctive. As a result, information from any competing ad would be separately stored with information from the focal ad in memory. Moreover, no information from the other ads is stored in memory. In such environments, consumers may easily recall information from any competing ad because they are less likely to confuse it with the focal ad and the other ads. Thus, the following hypothesis is proposed.

H2: The positive effect of ad distinctiveness on recall for any competing ad is more likely to occur in low competitive ad environments than in high competitive ad environments.

\section{Method}

\subsection{Design}

The hypotheses proposed in the previous section were tested using a 2 (distinctiveness of the focal ad: distinctive vs. undistinctive) $\times 2$ (competition level: high vs. low) between-subjects factorial design. Participants were 205 undergraduate students who took introductory marketing courses at a university in Tokyo. The average age was 19.7 years old and the proportion of males was $73.7 \%$. There were few significant differences in their memory performances because most of participants belonged to the same department and were nearly equal in terms of academic ability.

Participants were randomly assigned to four groups across the between-subjects factor, of which, participants in Group 1 were exposed to one distinctive focal ad and five competing ads (distinctive $\times$ high competitive), participants in Group 2 were exposed to one distinctive focal ad and one competing ad (distinctive $\times$ low competitive), participants in Group 3 were exposed to one undistinctive focal ad and five competing ads (undistinctive $\times$ high competitive), and participants in Group 4 were exposed to one undistinctive focal ad and one competing ad (undistinctive $\times$ low competitive).

\subsection{Stimuli}

\subsubsection{Product Categories and Brand Names}

Four types of product categories - tablet PCs, shoes, detergents, and candy-were selected based on FCB Grid (Vaughn 1980). The reason why we used a variety of products is to increase external validity. Regarding the brand names, a pretest was conducted to identify neutral brand names in terms of participants' prior knowledge or preferences (Keller 1991). As a result, six brand names were selected in 
each of the four product categories. These brand names are presented in Appendix 1.

\subsubsection{Advertisements}

Print ads were used because they afford greater experimental control (Keller 1987). In each of the four product categories, eight ads were created, which were composed of six undistinctive ads and two distinctive ads. Each undistinctive ad had a picture of a product in an upper half and copies (i.e., a headline, an introductory transition paragraph, and two additional paragraphs conveying product information) in a lower half (Keller 1987, 1991).

On the other hand, two types of distinctive focal ads - an ad with distinctive copies (i.e., consumers' comments about a product) or distinctive pictures (i.e., visually striking pictures) -were created. Three paid coders and the author discussed whether or not distinctiveness of the copies or the pictures were adequately manipulated. All ads for the experiment were revised based on advices from the coders.

\subsection{Procedure}

Each participant was exposed to the created print ads, of which each ad was presented in 30 seconds. Participants were asked to see ads as they did in their daily lives. The presentation order of ads was counterbalanced across participants to minimize serial position effects such as primacy and recency effects. Also, participants were not informed of which the focal ad for this experiment was.

After viewing all the ads, participants $\mathrm{re}^{-}$ sponded to questions. Finally, they were asked to describe everything they could remember about the focal ad as well as one of its competing ads, which was selected in advance by experimenter.

\subsection{Measures}

Responses to all items were given on a seven-point Likert scale from 1 (strongly disagree) to 7 (strongly agree). The items for distinctiveness of the focal ad developed for this study were: "I can distinguish the ad from others in terms of the ad copies/pictures," "I can understand the difference between the ad and others in the ad copies/pictures," and "The ad copies/pictures are dissimilar to others" ( $a=$ 0.88). The items for product category involvement were based on Keller (1987, 1991): "I have much knowledge about the product category," "I frequently purchase and/or use a brand in the product category," "Brand choice in the product category is important for me," and "I recognize differences in quality among brands" ( $a=0.76$ ). Recall was measured based on the total number of correct descriptions about the focal ad and its competing ad reported by each participant. 


\section{Results}

\subsection{Manipulation Checks}

Regarding distinctiveness of the focal ad, the results of the manipulation checks showed that participants significantly regarded the focal ad as more distinctive in the distinctive conditions than in the undistinctive conditions $(M=4.76$ vs. $2.20 ; t=11.42, p<0.01)$. Regarding the competition level, the results of the manipulation checks showed that recall was higher in the low competitive conditions than in the high competitive conditions in terms of both the focal ad $(M=2.26$ vs. $1.25 ; t=5.62, p<0.01)$ and the competing ad ( $M=1.93$ vs. 1.10 ; $t$ $=5.74, p<0.01)$. These data supported the intended manipulations.

\subsection{Tests of Hypothesis 1}

An analysis of covariance (ANCOVA) was conducted with distinctiveness of the focal ad and competition level as two explanatory variables, recall for the focal ad as explained varia-

〈Table 1〉 Means (SDs) of recall for the focal ad

\begin{tabular}{cccc}
\hline Competition level & Distinctiveness of the focal ad & Recall for the focal ad & Number of participants \\
\hline \multirow{2}{*}{ Low competitive } & Undistinctive & $\begin{array}{c}M=1.38 \\
(S D=1.16)\end{array}$ & $N=50$ \\
\cline { 2 - 4 } & Distinctive & $\begin{array}{r}M=2.71 \\
(S D=1.48)\end{array}$ & $N=52$ \\
\hline High competitive & Undistinctive & $\begin{array}{r}M=0.89 \\
(S D=0.83)\end{array}$ & $N=52$ \\
\cline { 2 - 4 } & Distinctive & $\begin{array}{c}M=1.44 \\
(S D=1.15)\end{array}$ & $N=51$ \\
\hline
\end{tabular}

〈Figure 1〉 The effect of ad distinctiveness on recall for the focal ad Recall for the focal ad

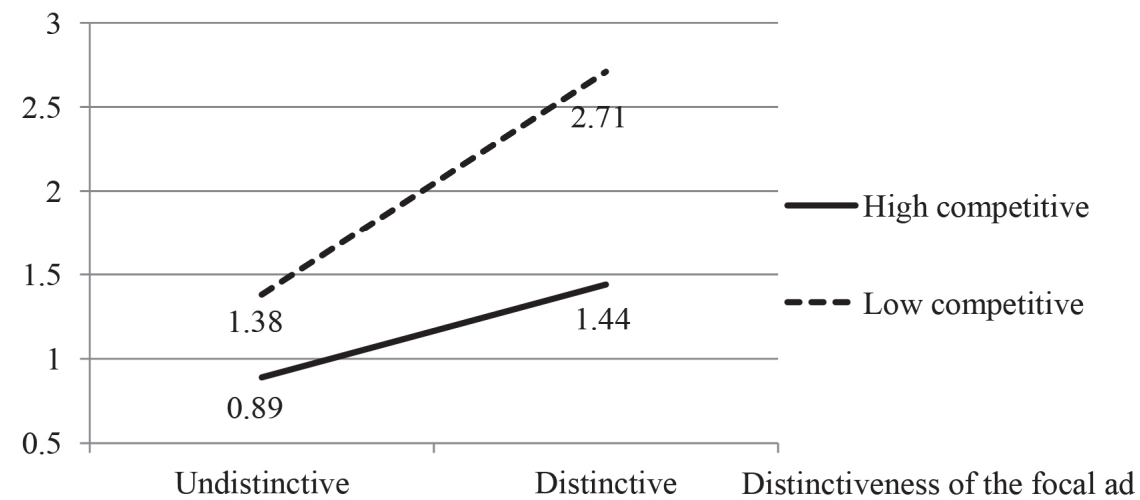


ble, and product category involvement as a covariate.

On recall for the focal ad, main effects of both ad distinctiveness $(F=35.52, p<0.01)$ and competition level $(F=26.41, p<0.01)$ were significant. The interaction effect was also significant $(F=4.33, p<0.05)$. In the high competitive conditions, recall for the distinctive focal ad ( $M=1.44)$ was higher than recall for the undistinctive focal ad $(M=0.89)$, suggesting a significant difference between the former and the latter $(F=4.69, p<0.05)$. In the low competitive conditions, recall for the distinctive focal ad $(M=2.71)$ was higher than recall for the undistinctive focal ad $(M=$ 1.38), suggesting a significant difference between the former and the latter $(F=26.04, p$ $<0.01)$. These results offered empirical support for $\mathrm{H} 1$.

\subsection{Tests of Hypothesis 2}

An analysis of covariance (ANCOVA) was conducted with distinctiveness of the focal ad and competition level as two explanatory variables, recall for any competing ad as explained

〈Table 2〉 Means (SDs) of recall for any competing ad

\begin{tabular}{cccc}
\hline Competition level & Distinctiveness of the focal ad & Recall for any competing ad & Number of Participants \\
\hline \multirow{2}{*}{ Low competitive } & $\begin{array}{r}M=1.32 \\
(S D=0.94)\end{array}$ & $N=50$ \\
\cline { 2 - 4 } & Undistinctive & $\begin{array}{r}M=2.23 \\
(S D=1.07)\end{array}$ & $N=52$ \\
\hline \multirow{2}{*}{ Distinctive } & $\begin{array}{r}M=0.91 \\
(S D=0.85)\end{array}$ & $N=52$ \\
\cline { 2 - 4 } & Undistinctive & $\begin{array}{c}M=1.19 \\
(S D=1.01)\end{array}$ & $N=51$ \\
\hline
\end{tabular}

〈Figure 2〉 The effect of ad distinctiveness on recall for any competing ad

Recall for any competing ad

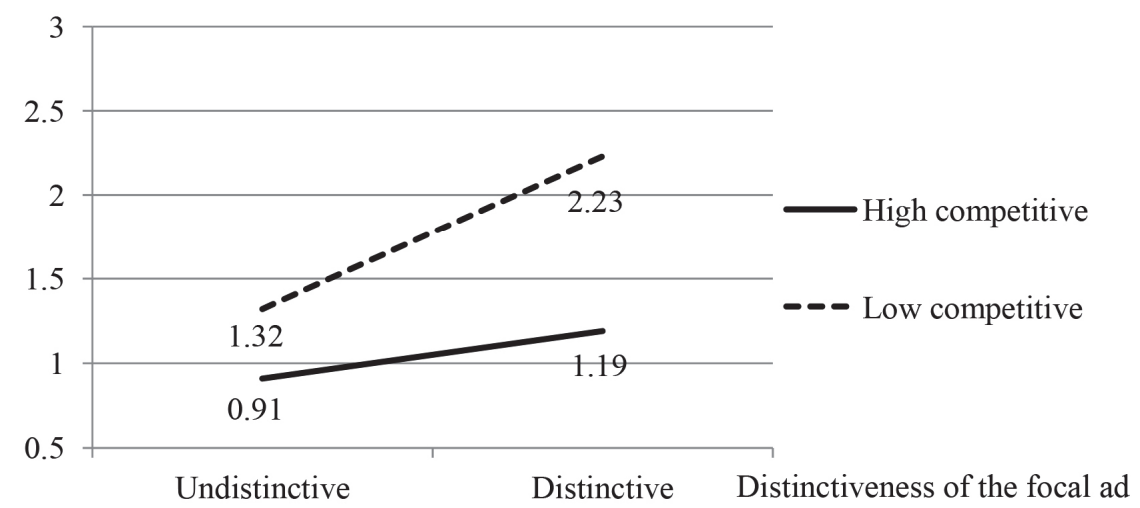


variable, and product category involvement as a covariate.

On recall for any competing ad, main effects of both ad distinctiveness $(F=35.52, p<$ $0.01)$ and competition level $(F=15.96, p<$ 0.01) were significant. The interaction effect was also significant $(F=4.26, p<0.05)$. In the high competitive conditions, there was no significant difference between recall for the competing ad in the presence of the distinctive focal ad and that in the presence of the undistinctive focal ad ( $M=1.19$ vs. $0.91 ; F=$ 1.87, $p>0.10)$. In the low competitive conditions, recall for the competing ad in the presence of the distinctive focal ad $(M=2.23)$ was higher than that in the presence of the undistinctive focal ad ( $M=1.32)$, suggesting a significant difference between the former and the latter $(F=18.35, p<0.01)$. These results offered empirical support for $\mathrm{H} 2$.

\section{Discussions}

\subsection{Theoretical Implications}

Previous studies on ad distinctiveness have claimed that when an advertiser makes its ad distinctive, recall for its own ad becomes higher in relatively less competitive ad environments (Keller 1991; Unnava and Sirdeshmukh 1994). In contrast, we found that ad distinctive- ness has positive effects on recall for the ad in both high and low competitive ad environments. Indeed, in high competitive environments, the positive effect of ad distinctiveness was relatively weak, but it is certain that ad distinctiveness enhanced recall for the ad.

Furthermore, while previous studies focused only on the relationship between ad distinctiveness and recall for the ad, we found that in low competitive ad environments, ad distinctiveness positively affected not only recall for the ad, but also recall for its competing ad.

\subsection{Managerial Implications}

Previous studies have found that ad repetition may not enhance recall for the ad in a high competitive market (e.g., Burke and Srull 1988; Malaviya et al. 1999). In contrast, our findings imply that ad differentiation can be an effective strategy to enhance recall for the ad even in a high competitive market. Thus, advertisers should make their ads distinctive rather than repeat their ads to reach target consumers.

Our findings also imply that in a low competitive market, when a competitor makes its ad distinctive, an ad may become distinctive automatically even if the advertiser does not try to make the ad distinctive. Therefore, an advertiser may be able to benefit from the competitor's ad and, thus, save its advertising expenditure. Conversely, it is necessary to pay attention to a risk that an advertiser may as- 
sist its competitor unintendedly.

\subsection{Limitations}

Several theoretical and empirical issues could not be resolved or even addressed in this one study. First, the experiment was conducted in a laboratory setting under which ad exposure was compressed and forced. Although participants were exposed to ads as they did in their daily lives, they might pay a higher level of attention to ads than usual. Other methods can address this limitation, but changing the methods may make it more difficult to manipulate ad distinctiveness adequately.

Second, in this study, we regarded one competing ad as the low competitive condition and five competing ads as the high competitive condition. The construct should be treated as a continuous variable to specify the point at which the positive effect of ad distinctiveness starts to decrease.

\subsection{Future Research}

Many opportunities exist for future research. First, we encourage researchers to analyze other explained variables: attitudes toward ads/brands (Laroche et al. 2006; Unnava and Sirdeshmukh 1994), intention to click (Yaveroglu and Donthu 2008), purchase intention (Laroche et al. 2006; Unnava and Sirdeshmukh 1994), and recognition (Malaviya et al. 1999). These additional analyses may lead to a better understanding of the effects of ad distinctiveness.

Second, the interaction between ad distinctiveness and ad repetition should be examined. As mentioned in the beginning of this article, many advertising researchers have focused on ad repetition that can reduce competitive interference though it has often been found that it does not enhance recall for the ad. Repetition of a distinctive ad may dramatically reduce competitive interference.

〈Received May 29. 2014〉 $\langle 1$ st Revised December 11. 2014〉 〈2nd Revised July 25. 2015〉 〈Accepted July 28. 2015〉

\section{References}

Anderson, Michael C. and James H. Neely (1996), "Interference and Inhibition in Memory Retrieval," in Bjork, Elizabeth L. and Robert A. Bjork eds., Memory, San Diego, CA: Academic Press, 237-313.

Blankenship, Albert B. and Paul L. Whitely (1941), "Proactive Inhibition in the Recall of Advertising Material," Journal of Social Psychology, 13 (May), 311-322.

Burke, Raymond R. and Thomas K. Srull (1988), "Competitive Interference and Consumer Memory for Advertising," Journal of Consumer Research, 15 (June), 55-68. 
Chunovic, Louis (2003), "Clutter Reaches AllTime High,” Television Week, 22 (May), 19. Creamer, Matthew (2007), "Caught in the Clutter Crossfire: Your Brand Why Pollution is Worsening despite Cleanup Efforts," Advertising Age, 78 (April), 1.

Danaher, Peter J., Andre Bonfrer, and Sanjay Dhar (2008), "The Effect of Competitive Interference on Sales for Packaged Goods," Journal of Marketing Research, 45 (April), 211-225.

Guerard, Katherine, Ian Neath, Aimee M. Surprenant, and Sebastien Tremblay (2010), "Distinctiveness in Serial Memory for Spatial Information," Memory \& Cognition, 38 (January), 83-91.

Hammer, Peter, Erica L. Riebe, and Rachel Kennedy (2009), "How Clutter Affects Advertising Effectiveness," Journal of $\mathrm{Ad}^{-}$ vertising Research, 49 (June), 159-163.

Jacoby, Larry and Fergus I. M. Craik (1979), "Effects of Elaboration of Processing at Encoding and Retrieval: Trace Distinctiveness and Recovery of Initial Context," in Cermak, Laird S. and Fergus I. M. Craik eds., Levels of Processing in Human Memory, Hillsdale, NJ: Erlbaum, 1-21.

Jeong, Yongick, Yeuseung Kim, and Xinshu Zhao (2011), "Competing for Consumer Memory in Television Advertising: An Empirical Examination of the Impacts of Non-Editorial Clutter on Brand Memory in Mega-Event Broadcasts," International
Journal of Advertising, 30 (4), 617-640. Keller, Kevin L. (1987), "Memory Factors in Advertising: The Effect of Advertising Retrieval Cues on Brand Evaluation,” Journal of Consumer Research, 14 (March), 316-333. (1991), "Memory and Evaluation Effects in Competitive Advertising Environments," Journal of Consumer Research, 17 (December), 463-476.

Laroche, Michel, Mark Cleveland, and Irene Maravelakis (2006), "Competitive Advertising Interference and Ad Repetition Effects: Comparing High-Share and Low-Share Brands," International Journal of Advertising, 25 (3), 271-307.

Malaviya, Prashant, Joan Meyers-Levy, and Brian Sternthal (1999), "Ad Repetition in a Cluttered Environment: The Influence of Type of Processing," Psychology \& Marketing, 16 (March), 99-118.

McKinney, Fred (1935), "Retroactive Inhibition in Advertising," Journal of Applied Psychology, 19 (June), 59-66.

Nelson-Field, Karen, Erica Riebe, and Byron Sharp (2013), "More Mutter about Clutter: Extending Empirical Generalizations to Facebook," Journal of Advertising Research, 53 (June), 186-191.

Riebe, Erica and John Dawes (2006), "Recall of Radio Advertising in Low and High Advertising Clutter Formats," International Journal of Advertising, 25 (1), 71-86.

Unnava, H. Rao and Deepak Sirdeshmukh (1994), 
"Reducing Competitive Ad Interference," Journal of Marketing Research, 31 (August), 403-411.

Vaughn, Richard (1980), "How Advertising Works: A Planning Model," Journal of Advertising Research, 20 (October), 27-33
Yaveroglu, Idil and Naveen Donthu (2008), "Advertising Repetition and Placement Issues in On-Line Environments," Journal of Advertising, 37 (March), 31-44. 


\section{〈Appendix 1〉 Brand names in each of the four product categories}

\begin{tabular}{|c|c|}
\hline Product categories & Brand names \\
\hline \multirow{6}{*}{ Tablet PCs } & Eee Pad Transformer \\
\hline & ICONIA TAB A500 \\
\hline & idea Pad Tablet A1 \\
\hline & Eee Slate B121 \\
\hline & ICONIA TAB A100 \\
\hline & ARROWS Tab LTE \\
\hline \multirow{6}{*}{ Shoes } & Suede Chukker Sneaker \\
\hline & $\begin{array}{l}\text { EWING ATLEITCS EWING } 33 \\
\text { HI RETRO wht / org-blu }\end{array}$ \\
\hline & $\begin{array}{c}\text { CLARKS WALLABEE ROCKY } \\
\text { MOUNTAIN FEATHERBED BROWN }\end{array}$ \\
\hline & NEW BALANCE M996GY MADE IN USA \\
\hline & $\begin{array}{l}\text { EWING ATLEITCS EWING } 33 \\
\text { HI RETRO red / blk }\end{array}$ \\
\hline & NIKE MAIN DRAW SL \\
\hline \multirow{6}{*}{ Detergents } & Snuggle EX \\
\hline & Arau \\
\hline & Hi-bec Zero \\
\hline & EMBRY \\
\hline & Ekitai Maruseru \\
\hline & Oxi Clean \\
\hline \multirow{6}{*}{ Candy } & 10 Assort Candy \\
\hline & Propolis Candy \\
\hline & Huwarinka Soft Candy (Beauty Rose Flavor) \\
\hline & Voice Care Nodoame \\
\hline & Dr. John's Fruit Hard Candy \\
\hline & Sawayaka Toiki Lemon Lime \\
\hline
\end{tabular}

